\title{
Dynamic Control Design Based on Limited Model Information
}

\author{
Farhad Farokhi and Karl H. Johansson
}

\begin{abstract}
The design of optimal $\mathrm{H}_{2}$ dynamic controllers for interconnected linear systems using limited plant model information is considered. Control design strategies based on various degrees of model information are compared using the competitive ratio as a performance metric, that is, the worst case control performance for a given design strategy normalized with the optimal control performance based on full model information. An explicit minimizer of the competitive ratio is found. It is shown that this control design strategy is not dominated by any other strategy with the same amount of model information. The result applies to a class of system interconnections and design information characterized through given plant, control, and design graphs.
\end{abstract}

\section{INTRODUCTION}

Many large-scale physical systems are composed of several smaller interconnected units. For these interconnected systems, it seems natural to employ local controllers which observe local states and control local inputs. The problem of designing such subcontrollers is usually addressed in the decentralized and distributed control literature [1][3]. Lately, there has been some efforts in formulating the problem of designing optimal decentralized controllers as a convex optimization problem for some specific classes of subsystem interconnection [4]-[8]. At the heart of all these decentralized and distributed control problems is the assumption that the control design is done with complete knowledge of the plant model. This is however not always possible in large-scale systems. It might be the case that (a) different subsystems belong to different individuals and they might be unwilling to share their model information since they may consider these information private, $(b)$ the design of each subcontroller is done by a different designer with no access to the global plant model since in the time of design the complete model information is not available, or (c) the designer is interested in designing each subcontroller using only local model information, so that the resulting subcontrollers do not need to be modified if the model parameters of a particular subsystem change over time. We call this special class of control design problems limited model information control design problems [9], [10]. In these problems, we assume that only some part of the plant model information is available to each subcontroller designer, but that the system interconnection structure and the common closed-loop cost function to be minimized are global knowledge.

F. Farokhi and K. H. Johansson are with ACCESS Linnaeus Center, School of Electrical Engineering, KTH-Royal Institute of Technology, SE10044 Stockholm, Sweden. E-mails: \{farokhi,kallej\}@ee.kth.se

The work of F. Farokhi and K. H. Johansson was supported by grants from the Swedish Research Council and the Knut and Alice Wallenberg Foundation.
The main contribution of this paper is to study the influence of the subsystem interconnection, the controller structure, and the amount of model information available to each subdesign on the closed-loop performance that a limited model information control design method can produce. We compare the control design methods using a performance metric called the competitive ratio, that is, the worst case control performance for a given design strategy normalized with the optimal control performance based on full model information. We find an explicit minimizer of the competitive ratio for a wide range of problems. Since this minimizer might not be unique, we show that it is also undominated, that is, there is no other control design method that acts always better while having the same worst-case ratio.

This paper is organized as follows. We formulate the problem of interest in Section II. We define a control design strategy and find its competitive ratio in Section III. In Section IV, we study the influence of interconnection pattern between different subsystems on the best limited model information control design method. We further study the achievable performance of limited model information design strategies when the controllers that they can produce are structured in Section V. The trade-off between the amount of plant information available to different parts of a control design strategy and the quality of controllers it can produce is considered in Section VI. Finally, we give the discussions on extensions in Section VII and end with the conclusions in Section VIII.

\section{A. Notation}

The sets of integer numbers, natural numbers, real numbers, and complex numbers are denoted respectively by $\mathbb{Z}$, $\mathbb{N}, \mathbb{R}$, and $\mathbb{C}$. The boundary of the unit circle in $\mathbb{C}$ is shown by $\mathbb{T}$. The space of Lebesgue measurable functions that are bounded on $\mathbb{T}$ is presented by $\mathcal{L}_{\infty}$ and $\mathcal{R} \mathcal{L}_{\infty}$ is the set of real proper rational transfer functions in $\mathcal{L}_{\infty}$. Additionally, all other sets are denoted by calligraphic letters such as $\mathcal{P}$ and $\mathcal{A}$.

Matrices are denoted by capital roman letters such as $A$. The entry in the $i^{\text {th }}$ row and the $j^{\text {th }}$ column of matrix $A$ is $a_{i j} . A_{j}$ will denote the $j^{\text {th }}$ row of $A$. $A_{i j}$ denotes a submatrix of matrix $A$, the dimension and the position of which will be defined in the text.

$A>(\geq) 0$ means that the symmetric matrix $A \in \mathbb{R}^{n \times n}$ is positive definite (positive semidefinite) and $A>(\geq) B$ means $A-B>(\geq) 0$. Let $\mathcal{S}_{++}^{n}\left(\mathcal{S}_{+}^{n}\right)$ be the set of symmetric positive definite (positive semidefinite) matrices in $\mathbb{R}^{n \times n}$.

All graphs considered in this paper are directed with vertex set $\{1, \ldots, q\}$ for a given $q \in \mathbb{N}$. All self-loops are present in 
the graphs that we consider in this paper, that is, $(i, i) \in E$ for all $1 \leq i \leq q$. We say that a vertex $i$ is a sink if there does not exist $j \neq i$ such that $(i, j) \in E$. The adjacency matrix $S \in\{0,1\}^{q \times q}$ of graph $G$ is a matrix whose entry $s_{i j}=1$ if $(j, i) \in E$ and $s_{i j}=0$ otherwise for all $1 \leq i, j \leq q$. In this paper, since the set of vertices is fixed for all the graphs, a subgraph of a graph $G$ is a graph whose edge set is a subset of the edge set of $G$ and a supergraph of a graph $G$ is a graph of which $G$ is a subgraph. We use the notation $G^{\prime} \supseteq G$ to indicate that $G^{\prime}$ is a supergraph of $G$.

$\underline{\sigma}(Y)$ and $\bar{\sigma}(Y)$ denote the smallest and the largest singular values of the matrix $Y$, respectively. Vector $e_{i}$ denotes the column vector with all entries zero except the $i^{\text {th }}$ entry which is equal to one. The function $\delta: \mathbb{Z} \rightarrow\{0,1\}$ is the unit-impulse function which is equal to one at origin and zero anywhere else.

\section{Problem Formulation}

\section{A. Plant Model}

Let a plant graph $G_{\mathcal{P}}$ with adjacency matrix $S_{\mathcal{P}}$ be given. Based on the adjacency matrix $S_{\mathcal{P}}$, we define the following set of matrices

$$
\begin{aligned}
\mathcal{A}\left(S_{\mathcal{P}}\right)=\left\{\bar{A} \in \mathbb{R}^{n \times n} \mid \bar{A}_{i j}=0 \in \mathbb{R}^{n_{i} \times n_{j}}\right. \text { for all } \\
\left.1 \leq i, j \leq q \text { such that }\left(s_{\mathcal{P}}\right)_{i j}=0\right\},
\end{aligned}
$$

where for each $1 \leq i \leq q, n_{i} \in \mathbb{N}$ is the order of subsystem $i$ and consequently $\sum_{i=1}^{q} n_{i}=n$. Besides, we define

$$
\begin{aligned}
\mathcal{B}(\epsilon)=\left\{\bar{B} \in \mathbb{R}^{n \times n} \mid \underline{\sigma}(\bar{B}) \geq \epsilon,\right. & \bar{B}_{i j}=0 \in \mathbb{R}^{n_{i} \times n_{j}} \\
& \text { for all } 1 \leq i \neq j \leq q\},
\end{aligned}
$$

for some given scalar $\epsilon>0$ and

$$
\begin{aligned}
\mathcal{H}=\left\{\bar{H} \in \mathbb{R}^{n \times n} \mid \operatorname{det}(\bar{H}) \neq 0,\right. & \bar{H}_{i j}=0 \in \mathbb{R}^{n_{i} \times n_{j}} \\
& \text { for all } 1 \leq i \neq j \leq q\} .
\end{aligned}
$$

Now we can introduce the set $\mathcal{P}$ of plants of interest as the space of all discrete-time linear time-invariant systems

$$
x(k+1)=A x(k)+B u(k)+H w(k) ; x(0)=0,
$$

with $A \in \mathcal{A}\left(S_{\mathcal{P}}\right), B \in \mathcal{B}(\epsilon)$, and $H \in \mathcal{H}$. With slightly abusing notation, we show a plant $P \in \mathcal{P}$ with triple $(A, B, H)$ since the set $\mathcal{P}$ is clearly isomorph to $\mathcal{A}\left(S_{\mathcal{P}}\right) \times \mathcal{B}(\epsilon) \times \mathcal{H}$. We will denote the ordered set of state indices related to subsystem $i$ with $\mathcal{I}_{i}$, that is, $\mathcal{I}_{i}:=\left(1+\sum_{j=1}^{i-1} n_{j}, \ldots, n_{i}+\right.$ $\left.\sum_{j=1}^{i-1} n_{j}\right)$. For subsystem $i$, state $\underline{x}_{i} \in \mathbb{R}^{n_{i}}$, control input $\underline{u}_{i} \in \mathbb{R}^{n_{i}}$, and exogenous input $\underline{w}_{i} \in \mathbb{R}^{n_{i}}$ are defined as

$$
\underline{x}_{i}=\left[\begin{array}{c}
x_{\ell_{1}} \\
\vdots \\
x_{\ell_{n_{i}}}
\end{array}\right], \underline{u}_{i}=\left[\begin{array}{c}
u_{\ell_{1}} \\
\vdots \\
u_{\ell_{n_{i}}}
\end{array}\right], \underline{w}_{i}=\left[\begin{array}{c}
w_{\ell_{1}} \\
\vdots \\
w_{\ell_{n_{i}}}
\end{array}\right]
$$

where the ordered set of indices $\left(\ell_{1}, \ldots, \ell_{n_{i}}\right) \equiv \mathcal{I}_{i}$, and its dynamic is specified by

$$
\underline{x}_{i}(k+1)=\sum_{j=1}^{q} A_{i j} \underline{x}_{j}(k)+B_{i i} \underline{u}_{i}(k)+H_{i i} \underline{w}_{i}(k) .
$$

An example of a plant graph $G_{\mathcal{P}}$ is given in Figure $1(a)$. For instance, the plant graph $G_{\mathcal{P}}$ shows that the second

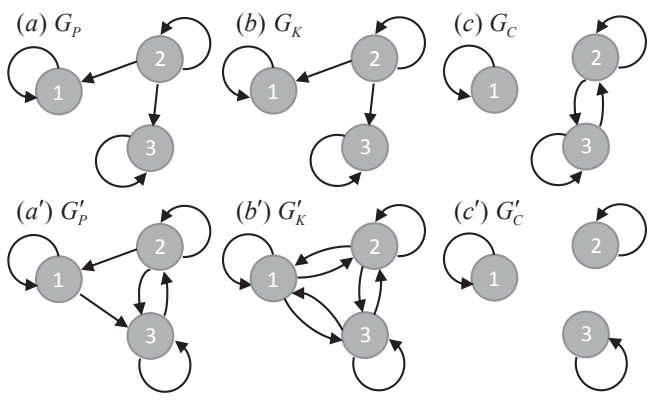

Fig. 1. $G_{\mathcal{P}}$ and $G_{\mathcal{P}}^{\prime}$ are examples of plant graphs, $G_{\mathcal{K}}$ and $G_{\mathcal{K}}^{\prime}$ are examples of control graphs, and $G_{\mathcal{C}}$ and $G_{\mathcal{C}}^{\prime}$ are examples of design graphs.

subsystem can affect the first and the third subsystems, that is, $A_{12}$ and $A_{32}$ can be nonzero. The first system is also a sink in the plant graph $G_{\mathcal{P}}$. An example of a plant graph $G_{\mathcal{P}}^{\prime}$ without sink is given in Figure $1\left(a^{\prime}\right)$.

\section{B. Controller}

Let a control graph $G_{\mathcal{K}}$ with adjacency matrix $S_{\mathcal{K}}$ be given. In this paper, we are interested in dynamic discretetime linear time-invariant state feedback control laws of the form

$$
\begin{gathered}
x_{K}(k+1)=A_{K} x_{K}(k)+B_{K} x(k) ; x_{K}(0)=0, \\
u(k)=C_{K} x_{K}(k)+D_{K} x(k),
\end{gathered}
$$

which can also be represented as the transfer function

$$
K \triangleq\left[\begin{array}{c|c}
A_{K} & B_{K} \\
\hline C_{K} & D_{K}
\end{array}\right]=C_{K}\left(z I-A_{K}\right)^{-1} B_{K}+D_{K},
$$

where $z$ is the symbol for one time-step forward shift operator. The controller $K$ must belong to

$$
\begin{aligned}
\mathcal{K}\left(S_{\mathcal{K}}\right)=\{\bar{K} & \in\left(\mathcal{R} \mathcal{L}_{\infty}\right)^{n \times n} \mid \bar{K}_{i j}=0 \in\left(\mathcal{R} \mathcal{L}_{\infty}\right)^{n_{i} \times n_{j}} \\
& \text { for all } \left.1 \leq i, j \leq q \text { such that }\left(s_{\mathcal{K}}\right)_{i j}=0\right\} .
\end{aligned}
$$

We refer to the set of controllers as $\mathcal{K}$ when adjacency matrix $S_{\mathcal{K}}$ can be deduced from the context or it is not relevant.

Figure 1(b) shows an example of an incomplete control graph $G_{\mathcal{K}}$ that characterizes a set of structured controllers. For instance, using control graph $G_{\mathcal{K}}$, we know that the third subsystem only has access to state measurements of the second subsystem beside its own state measurements, that is, $K_{31}=0$ while $K_{32}$ and $K_{33}$ can be nonzero.

\section{Control Design Methods}

A control design method $\Gamma$ is a map from the set of plants $\mathcal{P}$ to the set of controllers $\mathcal{K}$. Let a control design method $\Gamma$ be partitioned according to subsystems dimensions like

$$
\Gamma=\left[\begin{array}{ccc}
\Gamma_{11} & \cdots & \Gamma_{1 q} \\
\vdots & \ddots & \vdots \\
\Gamma_{q 1} & \cdots & \Gamma_{q q}
\end{array}\right]
$$

and a design graph $G_{\mathcal{C}}$ with adjacency matrix $S_{\mathcal{C}}$ be given. Each element $\Gamma_{i j}$ is a mapping $\mathcal{A}\left(S_{\mathcal{P}}\right) \times \mathcal{B}(\epsilon) \times \mathcal{H} \rightarrow$ $\left(\mathcal{R} \mathcal{L}_{\infty}\right)^{n_{i} \times n_{j}}$. We say that $\Gamma$ has structure $G_{\mathcal{C}}$ if, for all $1 \leq i \leq q$, the subsystem $i$ subcontroller is constructed with 
the knowledge of those subsystems $1 \leq j \leq q$ plant model such that $(j, i) \in E_{\mathcal{C}}$, that is, the mapping $\left[\Gamma_{i 1} \cdots \Gamma_{i q}\right]$ is only a function of $\left\{\left[A_{j 1} \cdots A_{j q}\right], B_{j j}, H_{j j} \mid\left(s_{\mathcal{C}}\right)_{i j} \neq 0\right\}$. The set of all these limited model information control design methods with structure $G_{\mathcal{C}}$ is denoted by $\mathcal{C}$.

Figure $1(c)$ shows an example of a design graph $G_{\mathcal{C}}$. For instance, using this design graph $G_{\mathcal{C}}$, we realize that the third subsystem model is available to the designer of the second subsystem controller but not the first subsystem model. Figure $1\left(c^{\prime}\right)$ illustrates an example of a fully disconnected design graph $G_{\mathcal{C}}^{\prime}$ with self-loops only which shows that the controller of all subsystems are constructed using only their own model information.

\section{Performance Metric}

The considered performance metrics is a modified version of the performance metrics originally defined in [9], [10]. Let us start with introducing the closed-loop performance measure.

To each plant $P=(A, B, H) \in \mathcal{P}$ and controller $K \in \mathcal{K}$, we associate a performance measure which is the $\mathrm{H}_{2}$ norm of the transfer function between the exogenous input $w(k)$ and the output

$$
y(k)=\left[\begin{array}{ll}
C^{T} 0 & 0
\end{array}{ }^{T} x(k)+\left[\begin{array}{ll}
0 & D^{T}
\end{array}\right]^{T} u(k),\right.
$$

where the matrices $C \in \mathbb{R}^{n \times n}$ and $D \in \mathbb{R}^{n \times n}$ are block diagonal full-rank matrices with each diagonal block entry belonging to $\mathbb{R}^{n_{i} \times n_{i}}$. Figure 2 illustrates the feedback system with the given controller $K$ and the overall-plant

$$
\hat{P}=\left[\begin{array}{c|cc}
A & H & B \\
\hline \hat{C} & 0 & \hat{D} \\
I & 0 & 0
\end{array}\right]
$$

where $\hat{C}=\left[\begin{array}{ll}C^{T} & 0\end{array}\right]^{T}$ and $\hat{D}=\left[\begin{array}{ll}0 & D^{T}\end{array}\right]^{T}$. Using the notation $\mathcal{F}(\hat{P}, K)$ for the closed-loop transfer function from $w(k)$ to $y(k)$, the performance measure can be written as

$$
J_{P}(K)=\|\mathcal{F}(\hat{P}, K)\|_{2} .
$$

We make the following standing assumption:

Assumption 2.1: $C=D=I$.

This is without loss of generality because the change of variables $(\bar{x}, \bar{u})=(C x, D u)$ transforms the output of the system and its state space representation into

$$
y(k)=\left[\begin{array}{ll}
I & 0
\end{array}\right]^{T} \bar{x}(k)+\left[\begin{array}{ll}
0 & I
\end{array}\right]^{T} \bar{u}(k),
$$

and

$$
\bar{x}(k+1)=C A C^{-1} \bar{x}(k)+C B D^{-1} \bar{u}(k) .
$$

This is done without changing the plant, control, or design graphs because of the block diagonal structure of matrices $C$ and $D$.

Definition 2.2: (Competitive Ratio) Let a plant graph $G_{\mathcal{P}}$, a control graph $G_{\mathcal{K}}$, and a constant $\epsilon>0$ be given. Let us assume that, for each plant $P \in \mathcal{P}$, there exists an optimal controller $K^{*}(P) \in \mathcal{K}$ such that

$$
J_{P}\left(K^{*}(P)\right) \leq J_{P}(K), \forall K \in \mathcal{K} .
$$

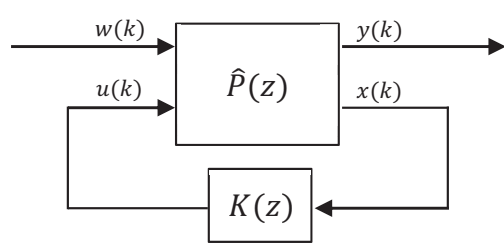

Fig. 2. The feedback system with the given controller $K$ and the overallplant $\hat{P}$.

The competitive ratio of a control design method $\Gamma$ is defined as

$$
r_{\mathcal{P}}(\Gamma)=\sup _{P=(A, B, H) \in \mathcal{P}} \frac{J_{P}(\Gamma(P))}{J_{P}\left(K^{*}(P)\right)}
$$

with the convention that " $\frac{0}{0}$ " equals one.

Definition 2.3: (Domination) A control design method $\Gamma^{\prime}$ is said to dominate another control design method $\Gamma$ if

$$
J_{P}\left(\Gamma^{\prime}(P)\right) \leq J_{P}(\Gamma(P)), \forall P=(A, B, H) \in \mathcal{P},
$$

with strict inequality holding for at least one plant in $\mathcal{P}$. When $\Gamma \in \mathcal{C}$ and no control design method $\Gamma^{\prime} \in \mathcal{C}$ exists that satisfies (4), we say that $\Gamma$ is undominated in $\mathcal{C}$.

\section{E. Mathematical Problem Formulation}

Now we can formulate the primary question concerning the connection between closed-loop performance and limited model information control design strategies. For a given plant graph $G_{\mathcal{P}}$, control graph $G_{\mathcal{K}}$, and design graph $G_{\mathcal{C}}$, we want to solve

$$
\arg \min _{\Gamma \in \mathcal{C}} r_{\mathcal{P}}(\Gamma)
$$

Since the solution to this problem might not be unique, we are interested in finding a minimizer that is also undominated. These solutions are the best worst-case designs with limited model information.

\section{PREliminary Results}

In order to give the main results of the paper, we need to define a control design strategy and find its competitive ratio.

Definition 3.1: Let a plant graph $G_{\mathcal{P}}$ and a constant $\epsilon>0$ be given. The control design method $\Gamma^{\Theta}$ is defined as

$$
\Gamma^{\Theta}(P)=-\operatorname{diag}\left(W_{1}(P), \ldots, W_{q}(P)\right) A,
$$

for all plants $P=(A, B, H) \in \mathcal{A}\left(S_{\mathcal{P}}\right) \times \mathcal{B}(\epsilon) \times \mathcal{H}$, where

$$
W_{i}(P)= \begin{cases}\left(I+B_{i i}^{T} X_{i i} B_{i i}\right)^{-1} B_{i i}^{T} X_{i i}, & \text { if } i \text { is a sink, } \\ B_{i i}^{-1}, & \text { otherwise, }\end{cases}
$$

and for each sink $i$ the matrix $X_{i i}$ is the unique positive definite solution of the discrete algebraic Riccati equation

$$
\begin{aligned}
A_{i i}^{T} X_{i i} A_{i i}-A_{i i}^{T} X_{i i} B_{i i}\left(I+B_{i i}^{T} X_{i i} B_{i i}\right)^{-1} B_{i i}^{T} X_{i i} A_{i i} & \\
& -X_{i i}+I=0 .
\end{aligned}
$$

The control design method $\Gamma^{\Theta}$ applies the so-called deadbeat strategy [10] to every subsystem that is not a sink (thus those closed-loop subsystems reach origin in just one timestep [11]) and, for every sink, applies the same optimal 
control law as if the node were decoupled from the rest of the graph.

LEMMA 3.2: The competitive ratio of the control design method $\Gamma^{\Theta}$ defined in (6) is $r_{\mathcal{P}}\left(\Gamma^{\Theta}\right)=\sqrt{1+1 / \epsilon^{2}}$ if one of the following conditions is satisfied:

(a) the plant graph $G_{\mathcal{P}}$ contains no isolated node and the control graph $G_{\mathcal{K}}$ is a complete graph;

(b) the acyclic plant graph $G_{\mathcal{P}}$ contains no isolated node and $G_{\mathcal{K}} \supseteq G_{\mathcal{P}}$.

Proof: Let $K_{C}^{*}(P)$ denotes the optimal static full-state feedback (centralized) controller for each plant $P \in \mathcal{P}$. According to the proof of the "only if" part of Theorem 3.6 in [10], we have

$$
Z \leq A^{T} B^{-T} B^{-1} A+I,
$$

for all plants $P=(A, B, H) \in \mathcal{P}$, where $Z$ is the unique positive definite solution of discrete algebraic Lyapunov equation

$$
\begin{aligned}
\left(A+B \Gamma^{\Theta}(P)\right)^{T} Z\left(A+B \Gamma^{\Theta}(P)\right)-Z & \\
& +I+\Gamma^{\Theta}(P)^{T} \Gamma^{\Theta}(P)=0 .
\end{aligned}
$$

Thus, the cost of the control design strategy $\Gamma^{\Theta}$ for each plant $P=(A, B, H)$ is upper-bounded as

$$
\begin{aligned}
J_{P}\left(\Gamma^{\Theta}(P)\right)^{2} & =\operatorname{tr}\left(H^{T} Z H\right) \\
& \leq \operatorname{tr}\left(H^{T}\left(A^{T} B^{-T} B^{-1} A+I\right) H\right) .
\end{aligned}
$$

where $\operatorname{tr}(\cdot)$ denotes the trace of a matrix. According to Theorem 3.2 in [10], it is evident that

$$
A^{T} B^{-T} B^{-1} A \leq\left(1+1 / \epsilon^{2}\right)(X-I),
$$

and equivalently

$$
\operatorname{tr}\left(H^{T} A^{T} B^{-T} B^{-1} A H\right) \leq\left(1+1 / \epsilon^{2}\right) \operatorname{tr}\left(H^{T}(X-I) H\right),
$$

where $X$ is the unique positive definite solution of discrete algebraic Riccati equation

$$
A^{T} X A-A^{T} X B\left(I+B^{T} X B\right)^{-1} B^{T} X A=X-I .
$$

Putting (10) in (9), we get

$$
\begin{aligned}
J_{P}\left(\Gamma^{\Theta}(P)\right)^{2} & \leq\left(1+1 / \epsilon^{2}\right) \operatorname{tr}\left(H^{T} X H\right) \\
& =\left(1+1 / \epsilon^{2}\right) J_{P}\left(K_{C}^{*}(P)\right)^{2} .
\end{aligned}
$$

Clearly, because $J_{P}\left(K_{C}^{*}(P)\right) \leq J_{P}\left(K^{*}(P)\right)$, irrespective of the control graph $G_{\mathcal{K}}$, we have

$$
J_{P}\left(\Gamma^{\Theta}(P)\right)^{2} \leq\left(1+1 / \epsilon^{2}\right) J_{P}\left(K^{*}(P)\right)^{2},
$$

and as a result

$$
r_{\mathcal{P}}\left(\Gamma^{\Theta}\right)=\sup _{P=(A, B, H) \in \mathcal{P}} \frac{J_{P}\left(\Gamma^{\Theta}(P)\right)}{J_{P}\left(K^{*}(P)\right)} \leq \sqrt{1+1 / \epsilon^{2}} .
$$

To show that this upper-bound is tight, we should exhibit plants for which it is attained.

Part a: Condition (a) is satisfied. Since there is no isolated node in the plant graph, we can pick indices $1 \leq i \neq j \leq q$ such that $\left(s_{\mathcal{P}}\right)_{i j} \neq 0$. The rest of the proof is given in two different cases.

Case a.1: Node $i$ is not a sink. Pick indices $i_{1} \in \mathcal{I}_{i}$ and $j_{1} \in \mathcal{I}_{j}$. Let $A(s)=s e_{i_{1}} e_{j_{1}}^{T}, B=\epsilon I$, and $H=I$. We get

$$
r_{\mathcal{P}}\left(\Gamma^{\Theta}\right) \geq \lim _{s \rightarrow \infty} \sqrt{\frac{s^{2} / \epsilon^{2}+n}{s^{2} /\left(1+\epsilon^{2}\right)+n}}=\sqrt{1+1 / \epsilon^{2}},
$$

since the unique positive definite solution of discrete algebraic Riccati equation in (11) is $X=I+\left[s^{2} /\left(1+\epsilon^{2}\right)\right] e_{j_{1}} e_{j_{1}}^{T}$, and as a result $J_{P}\left(K^{*}(P)\right)=\sqrt{s^{2} /\left(1+\epsilon^{2}\right)+n}$.

Case a.2: Node $i$ is a sink. We know $\left(s_{\mathcal{P}}\right)_{i i} \neq 0$ since all the self-loops are present. Pick $i_{1} \in \mathcal{I}_{i}$ and $j_{1} \in \mathcal{I}_{j}$. Let $A(r, s)=r e_{i_{1}} e_{i_{1}}^{T}+s e_{i_{1}} e_{j_{1}}^{T}, B=\epsilon I$, and $H=I$. According to Theorem 3.8 in [10], we get

$$
J_{P}\left(\Gamma^{\Theta}(P)\right)=\sqrt{\beta_{\Theta}\left(s^{2}+r^{2}\right)+n},
$$

where

$$
\beta_{\Theta}=\frac{\sqrt{r^{4}+2 r^{2} \epsilon^{2}-2 a r^{2}+\epsilon^{4}+2 \epsilon^{2}+1}+r^{2}-\epsilon^{2}-1}{2 \epsilon^{2} r^{2}} .
$$

Again, using Theorem 3.8 in [10], the optimal closed-loop performance is

$$
J_{P}\left(K^{*}(P)\right)=\sqrt{\beta_{K^{*}}\left(s^{2}+r^{2}\right)+n},
$$

where $\beta_{K^{*}}$ is

$$
\begin{aligned}
\beta_{K^{*}} & =\frac{\epsilon^{2} s^{2}+r^{2}\left(1+\epsilon^{2}\right)-\left(\epsilon^{2}+1\right)^{2}+\sqrt{c_{+} c_{-}}}{2 \epsilon^{2}\left(\epsilon^{2}+1\right)\left(s^{2}+r^{2}\right)}, \\
c_{ \pm} & =\epsilon^{2} s^{2}+\left(r^{2} \pm 2 r\right)\left(\epsilon^{2}+1\right)+\left(\epsilon^{2}+1\right)^{2} .
\end{aligned}
$$

Then, we get

$$
r_{\mathcal{P}}\left(\Gamma^{\Theta}\right) \geq \lim _{r \rightarrow \infty, \frac{s}{r} \rightarrow \infty} \frac{J_{P}\left(\Gamma^{\Theta}(P)\right)}{J_{P}\left(K^{*}(P)\right)}=\sqrt{1+1 / \epsilon^{2}} .
$$

Part b: Condition (b) is satisfied. Any acyclic directed graph has at least one sink. Let $i$ denote a sink in plant graph $G_{\mathcal{P}}$. Since there is no isolated node in the plant graph, there exists an index $j \neq i$ such that $\left(s_{\mathcal{P}}\right)_{i j} \neq 0$. Pick $i_{1} \in \mathcal{I}_{i}$ and $j_{1} \in \mathcal{I}_{j}$. Let $A(r, s)=r e_{i_{1}} e_{i_{1}}^{T}+s e_{i_{1}} e_{j_{1}}^{T}, B=\epsilon I$, and $H=I$. According to Lemma 4.1 in [12], we get

$$
J_{P}\left(K_{\mathcal{P}}^{*}(P)\right)=\sqrt{\beta_{K^{*}} s^{2}+\beta_{\Theta} r^{2}+n},
$$

where $K_{\mathcal{P}}^{*}(P)$ is the optimal controller when $G_{\mathcal{K}}$ is equal to $G_{\mathcal{P}}$. This results in

$$
\begin{aligned}
r_{\mathcal{P}}\left(\Gamma^{\Theta}\right) & \geq \lim _{r \rightarrow \infty, \frac{s}{r} \rightarrow \infty} \frac{J_{P}\left(\Gamma^{\Theta}(P)\right)}{J_{P}\left(K^{*}(P)\right)} \\
& \geq \lim _{r \rightarrow \infty, \frac{s}{r} \rightarrow \infty} \frac{J_{P}\left(\Gamma^{\Theta}(P)\right)}{J_{P}\left(K_{\mathcal{P}}^{*}(P)\right)}=\sqrt{1+1 / \epsilon^{2}}
\end{aligned}
$$

since clearly $J_{P}\left(K^{*}(P)\right) \leq J_{P}\left(K_{\mathcal{P}}^{*}(P)\right)$.

Lemma 3.2 shows that, if we apply the control design strategy $\Gamma^{\Theta}$ to a particular plant, the performance of the closed-loop system, at most, can be $\sqrt{1+1 / \epsilon^{2}}$ times the cost of the optimal control design strategy $K^{*}$.

There is no loss of generality in assuming that the plant graph $G_{\mathcal{P}}$ contains no isolated node since it is always 


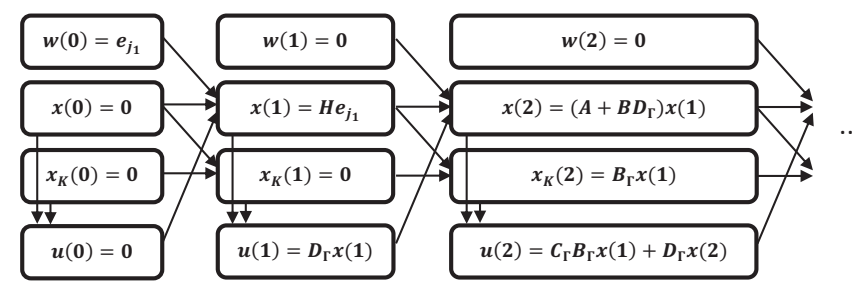

Fig. 3. State transition of the closed-loop system and its controller as a function of time for the exogenous input $w(k)=\delta(k) e_{j_{1}}$.

possible to design an optimal controller for an isolated subsystem without any model information about the other subsystems and without affecting them. In particular, this implies that there are $q \geq 2$ vertices in the plant graph.

\section{Plant Graph Influence on Achievable PERFORMANCE}

In this section, we study the achievable closed-loop performance, in terms of the competitive ratio and the domination, for different plant interconnection pattern. The next theorem shows that the control design strategy $\Gamma^{\Theta}$ is an undominated minimizer of the competitive ratio for all given plant graphs $G_{\mathcal{P}}$ when the control graph $G_{\mathcal{K}}$ is a complete graph and the design graph $G_{\mathcal{C}}$ is fully disconnected.

THEOREM 4.1: Let the plant graph $G_{\mathcal{P}}$ contain no isolated node, the control graph $G_{\mathcal{K}}$ be a complete graph, and the design graph $G_{\mathcal{C}}$ be a totally disconnected graph. Then, the competitive ratio of any control design strategy $\Gamma \in \mathcal{C}$ satisfies $r_{\mathcal{P}}(\Gamma) \geq r_{\mathcal{P}}\left(\Gamma^{\Theta}\right)$. Furthermore, the control design strategy $\Gamma^{\Theta}$ is undominated by set of limited model information control design strategies with design graph $G_{\mathcal{C}}$.

Proof: We use the following notation

$$
\Gamma(P)=\left[\begin{array}{l|l}
A_{\Gamma}(P) & B_{\Gamma}(P) \\
\hline C_{\Gamma}(P) & D_{\Gamma}(P)
\end{array}\right]
$$

to work with different parts of the state-space representation of a control design strategy $\Gamma$. The entries $A_{\Gamma}(P), B_{\Gamma}(P)$, $C_{\Gamma}(P)$, and $D_{\Gamma}(P)$ are matrices with appropriate dimension for each plant $P=(A, B, H) \in \mathcal{P}$. The matrices $A_{\Gamma}(P)$ and $C_{\Gamma}(P)$ are block diagonal matrices since different subcontrollers should not share state variables (each controller should be implemented in a decentralized fashion). This realization is not necessarily a minimal realization.

Consider indices $1 \leq i \neq j \leq q$ such that $\left(s_{\mathcal{P}}\right)_{i j} \neq 0$ (this is always possible since there is no isolated node in the plant graph). The rest of the proof is given in two different cases.

Case 1: Node $i$ is not a sink. Therefore, there exists an index $\ell \neq i$ such that $\left(s_{\mathcal{P}}\right)_{\ell i} \neq 0$. Pick indices $\ell_{1} \in \mathcal{I}_{\ell}$, $i_{1} \in \mathcal{I}_{i}$ and $j_{1} \in \mathcal{I}_{j}$ and define $A(r, s)=s e_{i_{1}} e_{j_{1}}^{T}+r e_{\ell_{1}} e_{i_{1}}^{T}$ and $B=\epsilon I$. Let $H_{j j}=r I$ and $H_{t t}=I$ for all $t \neq j$. Using the exogenous impulse input $w(k)=\delta(k) e_{j_{1}}$ and the time-steps given in Figure 3, we get

$$
\begin{aligned}
J_{P}(\Gamma(P))^{2} & \geq u_{\ell_{1}}(2)^{2}+x_{\ell_{1}}(3)^{2} \\
& =u_{\ell_{1}}(2)^{2}+\left(r^{2}\left(s+\epsilon\left(d_{\Gamma}\right)_{i_{1} j_{1}}(s)\right)+\epsilon u_{\ell_{1}}(2)\right)^{2} \\
& \geq r^{4}\left(s+\epsilon\left(d_{\Gamma}\right)_{i_{1} j_{1}}(s)\right)^{2} /\left(\epsilon^{2}+1\right),
\end{aligned}
$$

because, irrespective of the choice of $u_{\ell_{1}}(2)$, the function $u_{\ell_{1}}(2)^{2}+\left(r^{2}\left(s+\epsilon\left(d_{\Gamma}\right)_{i_{1} j_{1}}(s)\right)+\epsilon u_{\ell_{1}}(2)\right)^{2}$ is lower-bounded by $r^{4}\left(s+\epsilon\left(d_{\Gamma}\right)_{i_{1} j_{1}}(s)\right)^{2} /\left(\epsilon^{2}+1\right)$. It is worth mentioning that $\left(d_{\Gamma}\right)_{i_{1} j_{1}}(s)$ is only a function of the scalar $s$ and it is independent of the scalar $r$, since $r$ is in model parameters of subsystems $\ell, j \neq i$ and the design graph is fully disconnected. On the other hand

$$
\begin{aligned}
J_{P}\left(\Gamma^{\Delta}(P)\right) & =\sqrt{\operatorname{tr}\left(H^{T}\left(\left(1 / \epsilon^{2}\right) A^{T} A+I\right) H\right)} \\
& =\sqrt{\left(s^{2} r^{2}+r^{2}\right) / \epsilon^{2}+n-n_{j}+n_{j} r^{2}},
\end{aligned}
$$

where $\Gamma^{\Delta}$ is the deadbeat control design strategy and it is defined as $\Gamma^{\Delta}(P)=-B^{-1} A[10]$. Therefore

$$
\begin{aligned}
r_{\mathcal{P}}(\Gamma) & =\sup _{P \in \mathcal{P}} \frac{J_{P}(\Gamma(P))}{J_{P}\left(K^{*}(P)\right)} \\
& =\sup _{P \in \mathcal{P}}\left[\frac{J_{P}(\Gamma(P))}{J_{P}\left(\Gamma^{\Delta}(P)\right)} \frac{J_{P}\left(\Gamma^{\Delta}(P)\right)}{J_{P}\left(K^{*}(P)\right)}\right] \\
& \geq \sup _{P \in \mathcal{P}} \frac{J_{P}(\Gamma(P))}{J_{P}\left(\Gamma^{\Delta}(P)\right)} \\
& \geq \lim _{r \rightarrow \infty} \sqrt{\frac{r^{4}\left(s+\epsilon\left(d_{\Gamma}\right)_{i_{1} j_{1}}(s)\right)^{2} /\left(\epsilon^{2}+1\right)}{\left(s^{2} r^{2}+r^{2}\right) / \epsilon^{2}+n-n_{j}+n_{j} r^{2}}} .
\end{aligned}
$$

since $J_{P}\left(\Gamma^{\Delta}(P)\right) \geq J_{P}\left(K^{*}(P)\right)$ for all plants $P \in \mathcal{P}$. The competitive ratio $r_{\mathcal{P}}(\Gamma)$ is bounded only if $s+\epsilon\left(d_{\Gamma}\right)_{i_{1} j_{1}}(s)=$ 0 . Therefore, there is no loss of generality in assuming that $\left(d_{\Gamma}\right)_{i_{1} j_{1}}(s)=-s / \epsilon$ because otherwise the $r_{\mathcal{P}}(\Gamma)$ is infinity and the inequality $r_{\mathcal{P}}(\Gamma) \geq r_{\mathcal{P}}\left(\Gamma^{\Theta}\right)$ is trivially satisfied. Now, let us redefine $A(s)=s e_{i_{1}} e_{j_{1}}^{T}, H=I$ and $B=\epsilon I$. Since the parameters of the subsystem $i$ is not changed, we have $\left(d_{\Gamma}\right)_{i_{1} j_{1}}(s)=-s / \epsilon$. Therefore, for the same impulse exogenous input $w(k)=\delta(k) e_{j_{1}}$, we have

$$
J_{P}(\Gamma(P))^{2} \geq u_{i_{1}}(1)^{2}=\left(d_{\Gamma}\right)_{i_{1} j_{1}}(s)^{2}=s^{2} / \epsilon^{2},
$$

and

$$
r_{\mathcal{P}}(\Gamma) \geq \lim _{s \rightarrow \infty} \sqrt{\frac{s^{2} / \epsilon^{2}}{s^{2} /\left(1+\epsilon^{2}\right)+n}}=\sqrt{1+1 / \epsilon^{2}},
$$

since similar to Case $a .1$ in the proof of Lemma 3.2, we have $J_{P}\left(K^{*}(P)\right)=\sqrt{s^{2} /\left(1+\epsilon^{2}\right)+n}$.

Case 2: Node $i$ is a sink. We have $\left(s_{\mathcal{P}}\right)_{i i} \neq 0$ since all the self-loops are present. Let us pick $i_{1} \in \mathcal{I}_{i}$ and $j_{1} \in \mathcal{I}_{j}$. Let $A(r, s)=r e_{i_{1}} e_{i_{1}}^{T}+s e_{i_{1}} e_{j_{1}}^{T}, B=\epsilon I$, and $H=I$. According to the proof of the "only if" part of Theorem 3.6 in [10], for this particular family of plants, $\Gamma^{\Theta}(P)$ is the globally optimal $\mathrm{H}_{2}$ state-feedback controller. Now using Case $a .2$ in the proof of Lemma 3.2, it is easy to see that $r_{\mathcal{P}}(\Gamma) \geq \sqrt{1+1 / \epsilon^{2}}$.

To prove that the control design strategy $\Gamma^{\Theta}$ is undominated by set of limited model information control design strategies $\Gamma \in \mathcal{C}$, we construct plants $P=(A, B, H) \in \mathcal{P}$ that satisfy $J_{P}(\Gamma(P))>J_{P}\left(\Gamma^{\Theta}(P)\right)$ for any control design method $\Gamma \in \mathcal{C} \backslash\left\{\Gamma^{\Theta}\right\}$. The detailed proof of this part is given in [12].

As an example, consider the limited model information design problem given by the plant graph $G_{\mathcal{P}}^{\prime}$ in Figure $1\left(a^{\prime}\right)$, 
the control graph $G_{\mathcal{K}}^{\prime}$ in Figure $1\left(b^{\prime}\right)$, and the design graph $G_{\mathcal{C}}^{\prime}$ in Figure $1\left(c^{\prime}\right)$. Theorem 4.1 shows that the control design strategy $\Gamma^{\Theta}$ is the best control design strategy that one can propose based on the local model of subsystems since it is an undominated minimizer of the competitive ratio.

\section{Control Graph Influence on Achievable PERFORMANCE}

In this section, we study the structured controllers and their influence on the achievable closed-loop performance of the limited model information control design strategies. Note that finding the optimal control design strategy $K^{*}(P)$ is numerically intractable for general plant and control graphs. We use the results in [6], [7] which give an explicit solution to the problem of designing optimal decentralized controller for some special classes of subsystems interconnection and controller structures. Therefore, we assume that the plant graph $G_{\mathcal{P}}$ is an acyclic directed graph and the control graph $G_{\mathcal{K}}$ is a supergraph of the plant graph $G_{\mathcal{P}}$. Note that the control design strategy $\Gamma^{\Theta}$ is still applicable in this scenario.

THEOREM 5.1: Let the acyclic plant graph $G_{\mathcal{P}}$ contain no isolated node, the design graph $G_{\mathcal{C}}$ be a totally disconnected graph, and $G_{\mathcal{K}} \supseteq G_{\mathcal{P}}$. Then, the competitive ratio of any control design strategy $\Gamma \in \mathcal{C}$ satisfies $r_{\mathcal{P}}(\Gamma) \geq r_{\mathcal{P}}\left(\Gamma^{\Theta}\right)$. Furthermore, the control design strategy $\Gamma^{\Theta}$ is undominated by set of limited model information control design strategies with design graph $G_{\mathcal{C}}$.

Proof: Any acyclic directed graph has at least one sink. Let $i$ denote a sink in plant graph $G_{\mathcal{P}}$. Since there is no isolated node in the plant graph, there exists an index $j \neq$ $i$ such that $\left(s_{\mathcal{P}}\right)_{i j} \neq 0$. Pick $i_{1} \in \mathcal{I}_{i}$ and $j_{1} \in \mathcal{I}_{j}$. Let $A(r, s)=r e_{i_{1}} e_{i_{1}}^{T}+s e_{i_{1}} e_{j_{1}}^{T}, B=\epsilon I$, and $H=I$. According to the proof of the "only if" part of Theorem 3.6 in [10], for this particular family of plants, $\Gamma^{\Theta}(P)$ is the globally optimal $\mathrm{H}_{2}$ state-feedback controller. Now using Part $b$ of the proof of Lemma 3.2, it is easy to see that $r_{\mathcal{P}}(\Gamma) \geq \sqrt{1+1 / \epsilon^{2}}$.

The detailed proof of the part that control design strategy $\Gamma^{\Theta}$ is undominated is given in [12].

For instance, consider the limited model information design problem given by the plant graph $G_{\mathcal{P}}$ in Figure $1(a)$, the control graph $G_{\mathcal{K}}$ in Figure $1(b)$, and the design graph $G_{\mathcal{C}}^{\prime}$ in Figure $1\left(c^{\prime}\right)$. Theorem 5.1 illustrates that the control design strategy $\Gamma^{\Theta}$ is again the best control design strategy that one can propose based on the local model of subsystems, because it is an undominated minimizer of the competitive ratio.

\section{Design Graph Influence on ACHievable PERFORMANCE}

In this section, we try to determine the amount of the model information that we need in each subsystem to be able to setup a control design strategy $\Gamma$ with a smaller competitive ratio than the control design strategy $\Gamma^{\Theta}$.

THEOREM 6.1: Let the plant graph $G_{\mathcal{P}}$ and the design graph $G_{\mathcal{C}}$ be given and $G_{\mathcal{K}} \supseteq G_{\mathcal{P}}$. If the plant graph $G_{\mathcal{P}}$ contains the path $j \rightarrow i \rightarrow \ell$ with distinct vertices $i, j$, and $\ell$ while $(\ell, i) \notin E_{\mathcal{C}}$, then $r_{\mathcal{P}}(\Gamma) \geq r_{\mathcal{P}}\left(\Gamma^{\Theta}\right)$ for all $\Gamma \in \mathcal{C}$.
Proof: Because of the path $j \rightarrow i \rightarrow \ell$ with distinct vertices $i, j$, and $k$, we have $\left(s_{\mathcal{P}}\right)_{i j} \neq 0$ and $\left(s_{\mathcal{P}}\right)_{\ell i} \neq 0$. Pick indices $\ell_{1} \in \mathcal{I}_{\ell}, i_{1} \in \mathcal{I}_{i}$ and $j_{1} \in \mathcal{I}_{j}$ and define $A(r, s)=$ $s e_{i_{1}} e_{j_{1}}^{T}+r e_{\ell_{1}} e_{i_{1}}^{T}, B=\epsilon I$, and $H=I$. Similar to the proof of Theorem 4.1, using the exogenous impulse input $w(k)=$ $\delta(k) e_{j_{1}}$ and the time-steps given in Figure 3, we get

$$
J_{P}(\Gamma(P))^{2} \geq r^{2}\left(s+\epsilon\left(d_{\Gamma}\right)_{i_{1} j_{1}}(s)\right)^{2} /\left(\epsilon^{2}+1\right),
$$

Again, it should be noted that $\left(d_{\Gamma}\right)_{i_{1} j_{1}}(s)$ is only a function of the scalar $s$, and it is independent of the scalar $r$ because $r$ has appeared in model matrices of the subsystem $\ell \neq i$, and $(\ell, i) \notin E_{\mathcal{C}}$. We claim that for the competitive ratio to be bounded there should exist a positive constant $\theta \in \mathbb{R}$ independent of scalars $s$ such that $\left|s+\epsilon\left(d_{\Gamma}\right)_{i_{1} j_{1}}(s)\right| \leq \theta$. Assume this claim is not true, thus, there exist a sequence of scalars $\left\{s_{z}\right\}_{z=1}^{\infty} \subset \mathbb{R}$ such that

$$
\lim _{z \rightarrow \infty}\left|s_{z}+\epsilon\left(d_{\Gamma}\right)_{i_{1} j_{1}}\left(s_{z}\right)\right|=+\infty .
$$

Clearly, using (12) we get

$$
\begin{aligned}
r_{\mathcal{P}}(\Gamma) & \geq \lim _{z \rightarrow \infty, \frac{r}{s_{z}} \rightarrow \infty} \sqrt{\frac{r^{2}\left|s_{z}+\epsilon\left(d_{\Gamma}\right)_{i_{1} j_{1}}\left(s_{z}\right)\right|^{2} /\left(\epsilon^{2}+1\right)}{\left(s_{z}^{2}+r^{2}\right) / \epsilon^{2}+n}} \\
& =+\infty
\end{aligned}
$$

since $J_{P}\left(\Gamma^{\Delta}(P)\right)=\sqrt{\left(s_{z}^{2}+r^{2}\right) / \epsilon^{2}+n}$. Now, lets redefine $A(s)=s e_{i_{1}} e_{j_{1}}^{T}$. Since the model parameters of the subsystem $i$ is not changed, and its controller is not a function of the model parameters of subsystem $\ell$, the design entry $\left(d_{\Gamma}\right)_{i_{1} j_{1}}(s)$ stays the same. Therefore, $\left|s+\epsilon\left(d_{\Gamma}\right)_{i_{1} j_{1}}(s)\right| \leq \theta$ for all $s \in \mathbb{R}$, and as a result, for large enough $|s|$, we get $\left|\left(d_{\Gamma}\right)_{i_{1} j_{1}}(s)\right| \geq(|s|-\theta) / \epsilon$. Therefore, using the exogenous impulse input $w(k)=\delta(k) e_{j_{1}}$, we get

$$
J_{P}(\Gamma(P))^{2} \geq u_{i_{1}}(1)^{2}=\left(d_{\Gamma}\right)_{i_{1} j_{1}}(s)^{2} \geq(|s|-\theta)^{2} / \epsilon^{2},
$$

and

$$
r_{\mathcal{P}}(\Gamma) \geq \lim _{s \rightarrow \infty} \sqrt{\frac{(|s|-\theta)^{2} / \epsilon^{2}}{s^{2} /\left(1+\epsilon^{2}\right)+n}}=\sqrt{1+1 / \epsilon^{2}} .
$$

For this special plant, we know $K_{C}^{*}(P)=-\epsilon /\left(1+\epsilon^{2}\right) A$ belongs to the set $\mathcal{K}\left(S_{\mathcal{K}}\right)$ since the control graph $G_{\mathcal{K}} \supseteq$ $G_{\mathcal{P}}$, and consequently $J_{P}\left(K^{*}(P)\right) \leq J_{P}\left(K_{C}^{*}(P)\right)$ because $K^{*}(P)$ has a lower cost than any other controller is $\mathcal{K}\left(S_{\mathcal{K}}\right)$. On the other hand, clearly, for any plant $J_{P}\left(K_{C}^{*}(P)\right) \leq$ $J_{P}\left(K^{*}(P)\right)$. Therefore, for this special plant

$$
J_{P}\left(K^{*}(P)\right)=J_{P}\left(K_{C}^{*}(P)\right)=\sqrt{s^{2} /\left(1+\epsilon^{2}\right)+n} .
$$

This concludes the proof.

Consider the limited model information design problem given by the plant graph $G_{\mathcal{P}}^{\prime}$ in Figure $1\left(a^{\prime}\right)$, the control graph $G_{\mathcal{K}}^{\prime}$ in Figure $1\left(b^{\prime}\right)$, and the design graph $G_{\mathcal{C}}$ in Figure $1(c)$. Note that there is a path $3 \rightarrow 2 \rightarrow 1$ in the plant graph $G_{\mathcal{P}}$ but the edge $1 \rightarrow 2$ is not present in the design graph $G_{\mathcal{C}}$. Therefore, using Theorem 6.1, it is easy see that $r_{\mathcal{P}}(\Gamma) \geq r_{\mathcal{P}}\left(\Gamma^{\Theta}\right)$ for any $\Gamma \in \mathcal{C}$. 


\section{EXTENSIONS}

In this section, we relax the assumption that all the subsystems are required to be fully-actuated, that is, $B \in \mathcal{B}(\epsilon)$ is square invertible. To do so, we assume that plant graph $G_{\mathcal{P}}$ is an acyclic directed graph with $c \geq 1$ sinks since any acyclic graph has at least one sink. Accordingly, its adjacency matrix $S_{\mathcal{P}}$ is of the form

$$
S_{\mathcal{P}}=\left[\begin{array}{c|c}
\left(S_{\mathcal{P}}\right)_{11} & 0_{(q-c) \times(c)} \\
\hline\left(S_{\mathcal{P}}\right)_{21} & \left(S_{\mathcal{P}}\right)_{22}
\end{array}\right]
$$

where

$$
\begin{gathered}
\left(S_{\mathcal{P}}\right)_{11}=\left[\begin{array}{ccc}
\left(s_{\mathcal{P}}\right)_{11} & \cdots & \left(s_{\mathcal{P}}\right)_{1, q-c} \\
\vdots & \ddots & \vdots \\
\left(s_{\mathcal{P}}\right)_{q-c, 1} & \cdots & \left(s_{\mathcal{P}}\right)_{q-c, q-c}
\end{array}\right], \\
\left(S_{\mathcal{P}}\right)_{21}=\left[\begin{array}{ccc}
\left(s_{\mathcal{P}}\right)_{q-c+1,1} & \cdots & \left(s_{\mathcal{P}}\right)_{q-c+1, q-c} \\
\vdots & \ddots & \vdots \\
\left(s_{\mathcal{P}}\right)_{q, 1} & \cdots & \left(s_{\mathcal{P}}\right)_{q, q-c}
\end{array}\right],
\end{gathered}
$$

and $\left(S_{\mathcal{P}}\right)_{22}=\operatorname{diag}\left(\left(s_{\mathcal{P}}\right)_{q-c+1, q-c+1}, \ldots,\left(s_{\mathcal{P}}\right)_{q q}\right)$, where we assume, without loss of generality, that the vertices are numbered such that the sinks are labeled $q-c+1, \ldots, q$. We define the set $\mathcal{P}^{\prime}$ of plants of interest as the set of all triples $(A, B, H) \in \mathcal{A}\left(S_{\mathcal{P}}\right) \times \mathcal{B}^{\prime}(\epsilon) \times \mathcal{H}$ where

$$
\begin{aligned}
\mathcal{B}^{\prime}(\epsilon)=\left\{\bar{B} \in \mathbb{R}^{n \times m} \mid \underline{\sigma}(\bar{B}) \geq \epsilon,\right. & \bar{B}_{i j}=0 \in \mathbb{R}^{n_{i} \times m_{j}} \\
& \text { for all } 1 \leq i \neq j \leq q\} .
\end{aligned}
$$

Each $m_{i} \in \mathbb{N}$ is the number of control inputs in subsystem $i$, and consequently $\sum_{i=1}^{q} m_{i}=m$. Let relax $m_{i} \leq n_{i}$ for all $q-c+1 \leq i \leq q$ but force $m_{i}=n_{i}$ otherwise. In addition, all matrices $A$ and $B$ must satisfy

(a) $\left(A_{i i}, B_{i i}\right)$ is controllable,

(b) $\operatorname{span}\left(A_{i j}\right) \subseteq \operatorname{span}\left(B_{i i}\right)$ for all $j \neq i$ or equivalently there should exist a matrix $W_{i} \in \mathbb{R}^{m_{i} \times\left(n-n_{i}\right)}$ such that

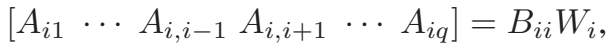

for all $q-c+1 \leq i \leq q$. For this new set of plants, the control design strategy $\Gamma^{\Theta}$ is still applicable since it does not require $B_{i i}$ to be invertible for $q-c+1 \leq i \leq q$.

Now we are ready to solve the problem (5) for this set of underactuated plants $\mathcal{P}^{\prime}$.

THEOREM 7.1: Let the acyclic plant graph $G_{\mathcal{P}}$ contain no isolated node, the control graph $G_{\mathcal{K}}$ be equal to the plant graph $G_{\mathcal{P}}$, and the design graph $G_{\mathcal{C}}$ be a totally disconnected graph. Then, the competitive ratio of any control design strategy $\Gamma \in \mathcal{C}$ satisfies $r_{\mathcal{P}}(\Gamma) \geq r_{\mathcal{P}}\left(\Gamma^{\Theta}\right)=\sqrt{1+1 / \epsilon^{2}}$ if $\left(S_{\mathcal{P}}\right)_{11}$ is not diagonal. Furthermore, the control design strategy $\Gamma^{\Theta}$ is undominated by set of limited model information control design strategies with design graph $G_{\mathcal{C}}$.

Proof: Similar to (14), we can write any $A \in \mathcal{A}\left(S_{\mathcal{P}}\right)$ as

$$
A=\left[\begin{array}{cc}
\tilde{A}_{11} & 0 \\
\tilde{A}_{21} & \tilde{A}_{22}
\end{array}\right] \text {, }
$$

where

$$
\tilde{A}_{11}=\left[\begin{array}{ccc}
A_{11} & \cdots & A_{1, q-c} \\
\vdots & \ddots & \vdots \\
A_{q-c, 1} & \cdots & A_{q-c, q-c}
\end{array}\right],
$$

$$
\tilde{A}_{21}=\left[\begin{array}{ccc}
A_{q-c+1,1} & \cdots & A_{q-c+1, q-c} \\
\vdots & \ddots & \vdots \\
A_{q 1} & \cdots & A_{q, q-c}
\end{array}\right],
$$

and $\tilde{A}_{22}=\operatorname{diag}\left(A_{q-c+1, q-c+1}, \ldots, A_{q q}\right)$. Clearly, if we apply deadbeat to all subsystems that are not sinks, the other subsystems (i.e., sinks) become decoupled (see Theorem 3.6 in [10]), and as a result

$$
\begin{aligned}
J_{P}\left(\Gamma^{\Theta}(P)\right)^{2}=J^{(1)}\left(\tilde{A}_{11}, \tilde{B}_{11},\right. & \left.\tilde{H}_{11}\right) \\
& +J^{(2)}\left(\tilde{A}_{21}, \tilde{A}_{22}, \tilde{B}_{22}, \tilde{H}_{22}\right)
\end{aligned}
$$

where $H=\operatorname{diag}\left(\tilde{H}_{11}, \tilde{H}_{22}\right), B=\operatorname{diag}\left(\tilde{B}_{11}, \tilde{B}_{22}\right)$, $J^{(1)}\left(\tilde{A}_{11}, \tilde{B}_{11}, \tilde{H}_{11}\right)$ is the cost of applying deadbeat control design to the nodes that are not sinks, and $J^{(2)}\left(\tilde{A}_{21}, \tilde{A}_{22}, \tilde{B}_{22}, \tilde{H}_{22}\right)$ is the cost of applying the same optimal control law as if the sinks were decoupled from the rest of the graph. Thus, we get

$$
J^{(1)}\left(\tilde{A}_{11}, \tilde{B}_{11}, \tilde{H}_{11}\right)=\operatorname{tr}\left(\tilde{H}_{11}^{T} \tilde{A}_{11}^{T} \tilde{B}_{11}^{-T} \tilde{B}_{11}^{-1} \tilde{A}_{11} \tilde{H}_{11}\right)
$$

and

$$
\begin{aligned}
J^{(2)}\left(\tilde{A}_{21}, \tilde{A}_{22}, \tilde{B}_{22}, \tilde{H}_{22}\right) & \leq \operatorname{tr}\left(\tilde{H}_{22}^{T} Y \tilde{H}_{22}\right) \\
& +\operatorname{tr}\left(\tilde{H}_{11}^{T} \tilde{A}_{21}^{T} \tilde{B}_{22}^{\dagger T} \tilde{B}_{22}^{\dagger} \tilde{A}_{21} \tilde{H}_{11}\right)
\end{aligned}
$$

where $\tilde{B}_{22}^{\dagger}=\left(B_{22}^{T} B_{22}\right)^{-1} B_{22}^{T}$. The inequality in (15) is true since $J^{(2)}\left(\tilde{A}_{21}, \tilde{A}_{22}, \tilde{B}_{22}, \tilde{H}_{22}\right)$ is the cost of the optimal control law as if the sinks were decoupled from the rest of the graph (see Theorem 3.6 in [10]), and it certainly has a lower cost than any other controller particularly

$$
K_{2}=-\left[\tilde{B}_{22}^{\dagger} \tilde{A}_{21}\left(I+\tilde{B}_{22}^{T} Y \tilde{B}_{22}\right)^{-1} \tilde{B}_{22}^{T} Y \tilde{A}_{22}\right],
$$

where $Y$ is the unique positive definite solution of discrete algebraic Riccati equation

$$
\begin{aligned}
\tilde{A}_{22}^{T} Y \tilde{A}_{22}-\tilde{A}_{22}^{T} Y \tilde{B}_{22}\left(I+\tilde{B}_{22}^{T} Y \tilde{B}_{22}\right)^{-1} \tilde{B}_{22}^{T} Y \tilde{A}_{22} & \\
& -Y+I=0 .
\end{aligned}
$$

Note that since $\tilde{A}_{22}$ is block diagonal, the positive definite matrix $Y$ is also block diagonal, and each block is only a function the corresponding subsystem. Thus, we get

$$
\begin{aligned}
& J_{P}\left(\Gamma^{\Theta}(P)\right)^{2} \leq \operatorname{tr}\left(\tilde{H}_{22}^{T} Y \tilde{H}_{22}\right)+ \\
& \quad \operatorname{tr}\left(\tilde{H}_{11}^{T}\left(\tilde{A}_{11}^{T} \tilde{B}_{11}^{-T} \tilde{B}_{11}^{-1} \tilde{A}_{11}+\tilde{A}_{21}^{T} \tilde{B}_{22}^{\dagger T} \tilde{B}_{22}^{\dagger} \tilde{A}_{21}\right) \tilde{H}_{11}\right) .
\end{aligned}
$$

The optimal closed-loop performance is $J_{P}\left(K^{*}(P)\right)^{2}=$ $\operatorname{tr}\left(H^{T} U H\right)$ where $U=\left[\begin{array}{ll}I_{n \times n} & 0\end{array}\right] V\left[\begin{array}{ll}I_{n \times n} & 0\end{array}\right]^{T}$ and $V$ is the unique positive definite solution of discrete algebraic Lyapunov equation in (17). The entries $A^{*}(P), B^{*}(P)$, $C^{*}(P)$, and $D^{*}(P)$ are state-space realization matrices of the optimal control design strategy $K^{*}(P)$ for a given plant $P \in \mathcal{P}^{\prime}$. Clearly, we have

$$
J_{P}\left(K^{*}(P)\right)^{2}=\sum_{t=1}^{n} e_{t}^{T} H^{T} U H e_{t}=\sum_{t=1}^{n} \sum_{k=0}^{\infty} y^{(t)}(k)^{T} y^{(t)}(k),
$$

where for each $t$ the vector $y^{(t)}(k)$ is the output of the system to the exogenous impulse input $w^{(t)}(k)=\delta(k) e_{t}$. This is true because for each $t$ the summation $\sum_{k=0}^{\infty} y^{(t)}(k)^{T} y^{(t)}(k)$ gives the diagonal element $e_{t}^{T} H^{T} U H e_{t}$. For any $P=$ 
$(A, B, H) \in \mathcal{P}^{\prime}$, we know that $H^{T} U H \geq H^{T} X H$ since centralized controller has the least performance cost over all other controllers either dynamic or static. Thus, for each $t \in \mathcal{N}=\bigcup_{z=1}^{q-c} \mathcal{I}_{z}$, we get $e_{t}^{T} H^{T} U H e_{t} \geq e_{t}^{T} H^{T} X H e_{t}$ which shows

$$
\sum_{t \in \mathcal{N}} \sum_{k=0}^{\infty} y^{(t)}(k)^{T} y^{(t)}(k) \geq \sum_{t \in \mathcal{N}} e_{t}^{T}\left(H^{T} X H\right) e_{t} .
$$

According to [13], we have $X \geq A^{T}\left(I+B B^{T}\right)^{-1} A+I$ for any $P \in \mathcal{P}^{\prime}$, and consequently

$$
\begin{aligned}
\sum_{t \in \mathcal{N}} \sum_{k=0}^{\infty} y^{(t)}(k)^{T} y^{(t)}(k) & \geq \operatorname{tr}\left(\tilde { H } _ { 1 1 } ^ { T } \left(\tilde{A}_{11}^{T}\left(I+\tilde{B}_{11} \tilde{B}_{11}^{T}\right)^{-1} \tilde{A}_{11}\right.\right. \\
+ & \left.\left.\tilde{A}_{21}^{T}\left(I+\tilde{B}_{22} \tilde{B}_{22}^{T}\right)^{-1} \tilde{A}_{21}\right) \tilde{H}_{11}\right) .
\end{aligned}
$$

On the other hand, for each $t \in \mathcal{S}=\bigcup_{z=q-c+1}^{q} \mathcal{I}_{z}$, we know there exists a sink $i$ such that $t \in \mathcal{I}_{i}$. For each $w^{(t)}(k)$, we get $\underline{x}_{j}=0$ for any $j \neq i$ (since $i$ is a sink in $\left.G_{\mathcal{P}}\right)$. The other subsystems cannot use state-measurements of subsystem $i$ because $G_{\mathcal{K}}$ is equal to $G_{\mathcal{P}}$ (and consequently $i$ is a sink in $G_{\mathcal{K}}$ ). Therefore, at best case scenario, the cost of controlling subsystem $i$ is equal to the cost of optimal controller designed locally (independent of other subsystems). Thus, we get

$$
\sum_{t \in \mathcal{S}} \sum_{k=0}^{\infty} y^{(t)}(k)^{T} y^{(t)}(k) \geq \operatorname{tr}\left(\tilde{H}_{22}^{T} Y \tilde{H}_{22}\right) .
$$

Therefore, we get

$$
\begin{aligned}
& J_{P}\left(K^{*}(P)\right)^{2} \geq \operatorname{tr}\left(\tilde { H } _ { 1 1 } ^ { T } \left(\tilde{A}_{11}^{T}\left(I+\tilde{B}_{11} \tilde{B}_{11}^{T}\right)^{-1} \tilde{A}_{11}\right.\right. \\
& \left.\left.\quad+\tilde{A}_{21}^{T}\left(I+\tilde{B}_{22} \tilde{B}_{22}^{T}\right)^{-1} \tilde{A}_{21}\right) \tilde{H}_{11}\right)+\operatorname{tr}\left(\tilde{H}_{22}^{T} Y \tilde{H}_{22}\right) .
\end{aligned}
$$

Now, lets define the set

$\mathcal{M}=\left\{\bar{\beta} \in \mathbb{R} \mid \bar{\beta} J_{P}\left(K^{*}(P)\right)-J_{P}\left(\Gamma^{\Theta}(P)\right) \geq 0 \forall P \in \mathcal{P}^{\prime}\right\}$.

Using the inequalities in (16) and in (18), it is evident if

$$
\begin{aligned}
& \operatorname{tr}\left(\tilde { H } _ { 1 1 } ^ { T } \left(\tilde{A}_{11}^{T}\left[\beta^{2}\left(I+\tilde{B}_{11} \tilde{B}_{11}^{T}\right)^{-1}-\tilde{B}_{11}^{-T} \tilde{B}_{11}^{-1}\right] \tilde{A}_{11}\right.\right. \\
+ & \left.\left.\tilde{A}_{21}^{T}\left[\beta^{2}\left(I+\tilde{B}_{22} \tilde{B}_{22}^{T}\right)^{-1}-\tilde{B}_{22}^{\dagger T} \tilde{B}_{22}^{\dagger}\right] \tilde{A}_{21}\right) \tilde{H}_{11}\right) \geq 0 .
\end{aligned}
$$

for some $\beta \in \mathbb{R}$, then $\beta$ would belong to $\mathcal{M}$. Thus, $\{\bar{\beta} \in$ $\left.\mathbb{R} \mid \bar{\beta} \geq \sqrt{1+1 / \epsilon^{2}}\right\} \subseteq \mathcal{M}$. This shows that $r_{\mathcal{P}}\left(\Gamma^{\Theta}\right) \leq$ $\sqrt{1+1 / \epsilon^{2}}$. Now if $\left(S_{\mathcal{P}}\right)_{11}$ is not diagonal, with the same argument as in the proof of Case 1 in Theorem 5.1, we get $r_{\mathcal{P}}(\Gamma) \geq r_{\mathcal{P}}\left(\Gamma^{\Theta}\right)=\sqrt{1+1 / \epsilon^{2}}$ for any $\Gamma \in \mathcal{C}$. This can be done because there are at least two fully-actuated subsystems and we can forget about the underactuated subsystems.

The proof of the part that the control design strategy $\Gamma^{\Theta}$ is undominated is similar to the one given in [12] for fullyactuated subsystems.

\section{CONCLUSIONS}

We considered optimal $\mathrm{H}_{2}$ dynamic control design for interconnected linear systems under limited plant model information. We introduced control design strategies as functions from the set of plants to the set of structured dynamic controller and compared these control design strategies using the competitive ratio as a performance metric. For a large class of system interconnections, controller structure, and design information, we found an explicit undominated minimizer of the competitive ratio.

\section{ACKNOWLEDGEMENTS}

The authors would like to thank Cédric Langbort for invaluable discussions, comments, and suggestions.

\section{REFERENCES}

[1] W. Levine, T. Johnson, and M. Athans, "Optimal limited state variable feedback controllers for linear systems," Automatic Control, IEEE Transactions on, vol. 16, no. 6, pp. 785-793, 1971.

[2] T. Söderström, "On some algorithms for design of optimal constrained regulators," Automatic Control, IEEE Transactions on, vol. 23, no. 6, pp. 1100-1101, 1978.

[3] B. Bamieh, F. Paganini, and M. Dahleh, "Distributed control of spatially invariant systems," Automatic Control, IEEE Transactions on, vol. 47, no. 7, pp. 1091-1107, 2002.

[4] M. Rotkowitz and S. Lall, "A characterization of convex problems in decentralized control," Automatic Control, IEEE Transactions on, vol. 51, no. 2, pp. 274-286, 2006.

[5] L. Lessard and S. Lall, "Internal quadratic invariance and decentralized control," in American Control Conference, Proceedings of the, pp. 5596-5601, 2010.

[6] J. Swigart and S. Lall, "Optimal synthesis and explicit state-space solution for a decentralized two-player linear-quadratic regulator," in Decision and Control, Proceedings of the 49th IEEE Conference on, pp. 132-137, 2010.

[7] P. Shah and P. Parrilo, " $\mathrm{H}_{2}$-optimal decentralized control over posets: A state space solution for state-feedback," in Decision and Control, Proceedings of the 49th IEEE Conference on, pp. 6722-6727, 2010.

[8] P. G. Voulgaris, "Optimal control of systems with delayed observation sharing patterns via input-output methods," Systems \& Control Letters, vol. 50, no. 1, pp. 51-64, 2003.

[9] C. Langbort and J.-C. Delvenne, "Distributed design methods for linear quadratic control and their limitations," Automatic Control, IEEE Transactions on, vol. 55, no. 9, pp. 2085-2093, 2010.

[10] F. Farokhi, C. Langbort, and K. H. Johansson, "Control design with limited model information," in American Control Conference, Proceedings of the, pp. 4697-4704, 2011.

[11] A. Emami-Naeini and G. Franklin, "Deadbeat control and tracking of discrete-time systems," Automatic Control, IEEE Transactions on, vol. 27, no. 1, pp. 176-181, 1982.

[12] F. Farokhi, C. Langbort, and K. H. Johansson, "Decentralized $\mathrm{H}_{2}$ control design with limited model information," Tech. Rep. TRITAEE 2011:064, 2011. https://eeweb01.ee.kth.se/ upload/publications/reports/2011/TRITA-EE 2011_064.pdf.

[13] N. Komaroff, "Iterative matrix bounds and computational solutions to the discrete algebraic Riccati equation," Automatic Control, IEEE Transactions on, vol. 39, no. 8, pp. 1676-1678, 1994. 